\title{
Effect of Alumina Morphology on the Clustering of Alumina Inclusions in Molten Iron
}

\author{
Lichun ZHENG, ${ }^{*}$ Annelies MALFLIET, Patrick WOLLANTS, Bart BLANPAIN and Muxing GUO \\ Department of Materials Engineering, KU Leuven, Kasteelpark Arenberg 44 Box 2450, 3001 Leuven, Belgium. \\ (Received on September 28, 2015; accepted on February 26, 2016; J-STAGE Advance published date: \\ April 21, 2016)
}

\begin{abstract}
Clustering of alumina inclusions during liquid processing of steel significantly influences its cleanliness and mechanical properties. We have therefore studied the effect of alumina inclusion morphology on their clustering behavior in molten iron. Alumina inclusions were extracted from iron samples taken at 1 min after Al addition. Dendritic, spherical, plate-like, faceted and clustered alumina inclusions were identified and their clustering degrees were measured. The clustering degree increases in the order of spherical, dendritic, plate-like and faceted inclusions. To explain this, attractive force between two alumina particles with different shape combinations, i.e., sphere-sphere (S-S), sphere-plane (S-P), plane-plane (P-P), was calculated based on the theory of spontaneous cavitation. The attractive force is influenced significantly by particle shape. S-S type has the smallest attractive force and the shortest acting length. P-P type has the largest attractive force and the longest acting length. This explains that spherical inclusions have the lowest clustering degree. The lower clustering degree of plate-like inclusions, compared with faceted inclusions, is due to that molten iron wets plate-like inclusions better.
\end{abstract}

KEY WORDS: alumina inclusions; interfacial properties; morphology; clustering degree; attractive force; cavitation.

\section{Introduction}

Clustering of non-metallic inclusions, especially of alumina inclusions, occurs in liquid steel during deoxidization. Due to clustering, fine inclusions grow rapidly, and thus float up more easily to the slag phase. However, large clusters may deteriorate the mechanical properties of steel when they remain in the solidified steel. ${ }^{1,2)}$ For this reason, maximum size of non-metallic inclusions is restricted in many steel grades. ${ }^{3)}$ Moreover, build-up of non-metallic inclusions on the submerged entry nozzles (SENs) may interrupt the continuous casting process, leading to decreased strand speed or premature replacement of SENs. ${ }^{4)}$ Clustering of inclusions in liquid steel and build-up of inclusions on the SENs both result from strong attractive forces between the inclusions. Due to SEN clogging, expensive countermeasures have to be taken, such as Ca-treatment. ${ }^{5,6)}$

Clustering of non-metallic inclusions in liquid steel has been intensively studied, mainly focusing on influencing factors, such as inclusion type, temperature, holding time, stirring and initial oxygen content. ${ }^{7-12)}$ Clustering was found to occur easily among inclusions that are not wetted by liquid steel. This suggests that clustering is related to the interfacial properties of inclusions and liquid steel. When inclusions are not wetted by liquid steel, withdrawal of liquid steel film between two approaching inclusions is energetically advan-

\footnotetext{
* Corresponding author: E-mail: zg.lichun@gmail.com DOI: http://dx.doi.org/10.2355/isijinternational.ISIJINT-2015-561
}

tageous, facilitating the clustering process. When inclusions are wetted by liquid steel, liquid steel film stays between the inclusions and inhibits clustering. Clustering of inclusions both on liquid steel surface and at slag/steel interfaces has been extensively studied. With confocal scanning laser microscopy (CSLM), unwetted inclusions are observed to move to each other, followed by collision and agglomeration. ${ }^{13-19)}$ The attractive force is considered to arise from the capillary force, which is due to the deformation of the melt surface around the inclusions. ${ }^{16,20)}$ In the bulk of liquid steel, however, studies of clustering of inclusions are still limited from the viewpoint of interfacial properties. Yaminsky et $a .^{21,22)}$ proposed that liquid may be spontaneously expelled from the region between two approaching particles unwetted by the liquid to form a gas cavity around the contact zone, resulting in a strong attractive force. Cournil et al. ${ }^{23,24)}$ reported that unwetted particles can form bigger clusters and that fragmentation of the clusters happens less significantly, compared with wetted particles. Nakamoto et al. ${ }^{25)}$ compared neck growth of alumina balls at sintering in molten iron with that in Ar atmosphere and found that neck growth in molten iron proceeds much faster. After analyzing influencing factors, they concluded that the fast neck growth is not caused by the interfacial energy, but by the non-wetting property, which induces the formation of a gas cavity between the two alumina balls. Sasai et al. ${ }^{26)}$ found that the clustering of alumina inclusions is promoted when molten $\mathrm{FeO}$ is present in liquid steel and concluded that molten $\mathrm{FeO}$ forms a liquid bridge between alumina inclusions and serves 
as a binder during the clustering. Such a conclusion was also obtained by Mizoguchi et al. $^{27)}$ after comparing iron content in alumina inclusions sampled from liquid steel after $\mathrm{RH}$-degassing and from a cast slab. However, molten $\mathrm{FeO}$ is thermodynamically unstable in Al-killed steel. Therefore, this conclusion should be reconsidered carefully. Recently, Sasai ${ }^{28)}$ directly measured the attractive force between two alumina cylinders $(D=8 \mathrm{~mm}, H=30 \mathrm{~mm})$ in molten iron with a novel method and confirmed that the strong attractive force derives from a gas cavity between the two cylinders not from the van der Waals force. Based on the above literature studies, it can be concluded that the cavitation theory has attracted more and more attention to interpret the clustering phenomena of non-metallic inclusions in liquid steel. To the best of our knowledge, however, studies of the clustering of non-metallic inclusions with this theory are still limited, especially focusing on the effects of wettability, surface tension of the melt, particle size and shape.

This paper is a follow-up study of the effect of interfacial properties of alumina inclusions and molten iron on the clustering of alumina inclusions. To modify the interfacial properties, Te, which is a strong surface active element in molten iron, was added to molten iron before Al deoxidization. In our previous paper, ${ }^{29)}$ we found that the extent of clustering increases with Te addition from 0 to $250 \mathrm{ppm}$ and thereafter decreases with further Te addition especially in the samples taken at $10 \mathrm{~min}$ after $\mathrm{Al}$ addition. To explain this, we proposed that the clustering of alumina inclusions has a close relationship with alumina morphology, which is modified considerably due to Te addition.

In the present work, we focus on the effect of alumina morphology on the clustering of alumina inclusions. Alumina inclusions were extracted from iron matrix and observed with a high resolution SEM. Clustering degrees of spherical, dendritic, plate-like and faceted inclusions were measured, respectively. The effect of alumina morphology on the clustering is quantitatively analyzed based on the theory of spontaneous cavitation between unwetted particles. Influencing factors on the clustering of alumina inclusions, such as wettability, surface tension of melt and particle size, are also discussed.

\section{Experimental Methods}

\subsection{Procedure}

A detailed description of the experiments is given in our previous paper. ${ }^{29)}$ The experiments were carried out in a vertical tube furnace (heating element $\mathrm{MoSi}_{2}$ ) under purified $\mathrm{Ar}$ atmosphere (flowrate $0.5 \mathrm{~L} / \mathrm{min}, \mathrm{Po}_{2}<10^{-20} \mathrm{~atm}$ ). $80 \mathrm{~g}$ of electrolytic iron $(99.99 \%$, Mairon SHP, Toho Zinc Co. Ltd.) together with reagent grade $\mathrm{Fe}_{2} \mathrm{O}_{3}$ powder used to adjust the initial oxygen content was filled in a magnesia crucible (27 mm ID and $50 \mathrm{~mm} \mathrm{H}$ ) and melted at $1873 \mathrm{~K}$. After homogenizing for $30 \mathrm{~min}$, Te was added through a

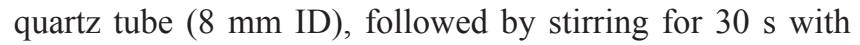
an alumina rod. 3 min later, Al (99.99\%) was added in the same way, followed by stirring for 0 or $30 \mathrm{~s}$. Then, the melt was held at $1873 \mathrm{~K}$. Iron samples were taken with a quartz tube (6 mm ID) at 1,3 , and $10 \mathrm{~min}$ after $\mathrm{Al}$ addition, and rapidly quenched in water. Detailed experimental conditions are listed in Table 1. The additions of 3.2, 20 and $80 \mathrm{mg}$ Te
Table 1. Details of the experimental conditions.

\begin{tabular}{ccccc}
\hline Test No. & $\mathrm{Fe}_{2} \mathrm{O}_{3}$ & $\mathrm{Te}$ & $\mathrm{Al}$ & Stirring \\
\cline { 2 - 5 } & $(\mathrm{g})$ & $(\mathrm{mg})$ & $(\mathrm{g})$ & $(\mathrm{s})$ \\
\hline L1 & 0 & 0 & 0.08 & 0 \\
L2 & & 80 & & \\
LS1 & & 0 & 0.08 & 30 \\
LS2 & 0 & 80 & & \\
HS1 & & 0 & & 30 \\
HS2 & & 3.2 & 0.13 & \\
HS3 & 0.11 & 20 & & \\
HS4 & & 80 & & \\
\hline
\end{tabular}

to molten iron correspond to 40, 250 and $1000 \mathrm{ppm}$ Te in molten iron, respectively.

\subsection{Observation of Inclusion Morphology}

$0.1 \mathrm{~g}$ of iron was dissolved in a $\mathrm{HCl}-\mathrm{HNO}_{3}$ acid solution $\left(\mathrm{HCl} / \mathrm{HNO}_{3} / \mathrm{H}_{2} \mathrm{O}=10 / 1 / 10\right)$ at $90^{\circ} \mathrm{C}$. After complete dissolution $(4 \mathrm{~h})$, the solution was filtered on a polycarbonate membrane with $0.2 \mu \mathrm{m}$ pore size. The membrane was washed, dried and coated with $\mathrm{Au}-\mathrm{Pd}$. Alumina inclusions on the membrane were subjected to SEM (Philips XL 30 FEG) observation. Successive micrographs were taken at a magnification of 5000 . For each sample, more than 500 particles with a diameter larger than $0.8 \mu \mathrm{m}$ were measured for studying morphology distribution and clustering tendency of alumina inclusions.

\section{Results and Discussion}

\subsection{Morphology Distribution of Alumina Inclusions}

During SEM observation, dendritic, spherical, faceted, plate-like and clustered alumina inclusions were identified. The classification in the present work follows that of Braun et $a l .{ }^{11)}$ Dendritic inclusions develop with a typical multibranching form; spherical inclusions are singular and in the shape of sphere; faceted inclusions have well-developed crystal faces and are three-dimensional; plate-like inclusions also have well-developed crystal faces but appear as twodimensional, showing a platy $\{222\}$ form; clusters consist of two or more individual particles of the above mentioned morphologies. Figure 1 shows the representative morphologies of the inclusions observed in this work. The origin of different morphologies of alumina inclusions in liquid steel has been reviewed by Dekkers et al. ${ }^{30}$

The morphology of a crystal is determined intrinsically by its internal structure. ${ }^{31)}$ External factors, such as supersaturation degree, holding time, temperature, stirring, impurity and solvent, can cause large changes to the growth rates of individual faces and hence modify the morphology. ${ }^{31)}$ In the present work, an obvious effect of impurity Te on the morphology of alumina inclusions was observed. To evaluate the extent of morphological modification by Te, morphology distribution of alumina inclusions was measured after acid extraction. The morphology distribution represents the relative frequency of dendritic, spherical, plate-like and faceted inclusions. Particles in clusters were also included in the 

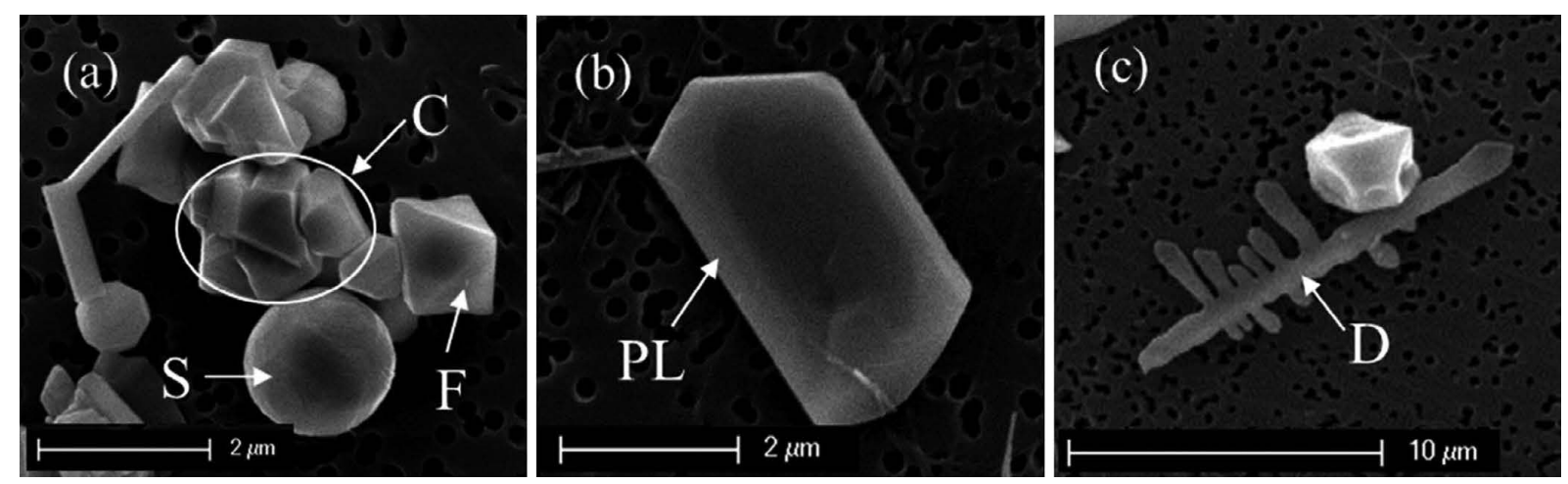

Fig. 1. Alumina morphologies after acid extraction: spherical (S), dendritic (D), faceted (F), plate-like (PL) and clustered $(\mathrm{C})$

Table 2. Morphology distribution of alumina inclusions in samples taken at $1 \mathrm{~min}$ after $\mathrm{Al}$ addition.

\begin{tabular}{crrccc}
\hline \multirow{2}{*}{$\begin{array}{c}\text { Sample } \\
\text { No. }\end{array}$} & Te (ppm) & \multicolumn{4}{c}{ Morphology distribution/\% } \\
\cline { 3 - 6 } & & Spherical & Dendritic & Faceted & Plate-like \\
\hline L1 & 0 & 68.0 & 1.6 & 21.6 & 8.8 \\
L2 & 1000 & 33.9 & 3.6 & 32.1 & 30.5 \\
LS1 & 0 & 23.7 & 4.2 & 55.4 & 16.7 \\
LS2 & 1000 & 4.0 & 6.2 & 43.2 & 46.6 \\
HS1 & 0 & 3.1 & 19.7 & 66.0 & 11.2 \\
HS2 & 40 & 3.7 & 4.7 & 78.2 & 13.5 \\
HS3 & 200 & 4.8 & 4.1 & 75.9 & 15.2 \\
HS4 & 1000 & 8.3 & 3.0 & 68.1 & 20.6 \\
\hline
\end{tabular}

distribution. Since long holding time facilitates sintering and densification of clustered particles, leading to difficulties in determining the number and morphology of individual alumina particles in clusters, only samples taken at $1 \mathrm{~min}$ after $\mathrm{Al}$ addition were analyzed. The results were shown in Table 2.

It is clear that Te considerably modifies the morphology of alumina inclusions. Te tends to make the morphology of alumina inclusions evolve from dendritic or spherical, via faceted, to plate-like. The effectiveness of Te on the morphological modification closely relies on the experimental conditions. Stirring strengthens the effectiveness of Te, resulting in more plate-like inclusions. On the contrary, increasing the supersaturation degree expressed as $\left(a_{\mathrm{Al}}^{2 / 3} \cdot a_{\mathrm{O}}\right) /\left(a_{\mathrm{Al}}^{2 / 3} \cdot a_{\mathrm{O}}\right)_{\mathrm{eq}}$ weakens the effectiveness of $\mathrm{Te}$, decreasing the amount of plate-like inclusions. Mechanism of Te action on the morphology of alumina inclusions will be studied in a following paper.

\subsection{Clustering Degree of Different Morphologies of Alumina Inclusions}

To study the extent of clustering of alumina inclusions, clustering degree $C_{\mathrm{D}}$ is defined as the ratio of the number of clustered particles to the total number of particles. The $C_{\mathrm{D}}$ values for the samples taken at $1 \mathrm{~min}$ after $\mathrm{Al}$ addition are listed in the rightest column of Table 3. It was found that Te considerably enhances the clustering of alumina inclusions. This is consistent with 2D measurements in our
Table 3. Clustering degree of different types of alumina inclusions.

\begin{tabular}{cccccc}
\hline \multirow{2}{*}{$\begin{array}{c}\text { Sample } \\
\text { No. }\end{array}$} & \multicolumn{5}{c}{ Clustering degree/\% } \\
\cline { 2 - 6 } & Spherical & Dendritic & Plate-like & Faceted & Overall \\
\hline L1 & 9.9 & 28.6 & 55.3 & 71.0 & 27.4 \\
L2 & 16.6 & 38.9 & 64.5 & 77.5 & 51.6 \\
\hline LS1 & 6.0 & 20.0 & 35.6 & 68.4 & 46.1 \\
LS2 & 23.1 & 15.0 & 24.5 & 72.1 & 44.4 \\
\hline HS1 & 14.3 & 33.8 & 51.3 & 83.5 & 68.0 \\
HS2 & 20.0 & 50.0 & 64.6 & 87.2 & 80.0 \\
HS3 & 32.5 & 42.9 & 72.3 & 94.2 & 85.8 \\
HS4 & 29.3 & 47.6 & 72.2 & 92.0 & 81.4 \\
\hline
\end{tabular}

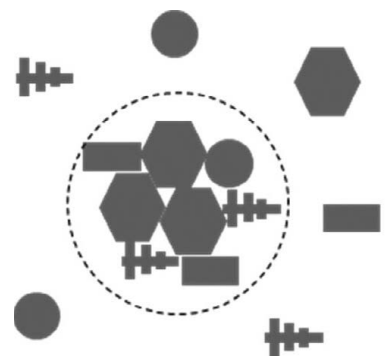

Spherical: $C_{\mathrm{D}}=1 / 3$

Dendritic: $C_{\mathrm{D}}=2 / 4$

Plate-like : $C_{\mathrm{D}}=2 / 3$

Faceted: $C_{\mathrm{D}}=3 / 4$

Overal : $C_{\mathrm{D}}=8 / 14$

Fig. 2. Schematic illustration of determining the clustering degree of alumina inclusions.

previous paper. ${ }^{29)}$ To clarify whether this enhancement is related to the morphological modification of the inclusions shown in Table 2, clustering degree of each type of alumina inclusions was measured. The measurement approach is schematically illustrated in Fig. 2. The results are presented both in Fig. 3 and Table 3. For a high reliability of the results, more than 500 particles in each sample were carefully analyzed with respect to their morphologies.

Figure 3(a) shows the $C_{\mathrm{D}}$ results of samples L1 and L2. In both samples, the $C_{\mathrm{D}}$ values increase in the order of spherical, dendritic, plate-like and faceted inclusions. The same phenomenon was also observed in samples LS1, LS2 and HS1 to HS4, as shown in Figs. 3(b) and 3(c). Figure 4 shows the average clustering degree of each type of alumina inclusions based on the results of samples HS1 to HS4. The 
error bars represent the lowest and the highest clustering degrees in the samples. The average $C_{\mathrm{D}}$ values are $24.0 \%$, $43.6 \%, 65.1 \%$ and $89.2 \%$ for spherical, dendritic, plate-like and faceted inclusions, respectively. These results show that faceted inclusions have the highest tendency of forming clusters while spherical inclusions have the lowest tendency, as seen clearly from the SEM images in Fig. 5. Similar results were also noticed by Braun et al., ${ }^{11)}$ who found that the extent of clustering of alumina inclusions in high oxygen ingots, where the inclusions are mostly spherical, is much less than in low oxygen ingots, where the inclusions are predominantly faceted. Compared with spherical inclusions, the higher clustering degree of dendritic inclusions is due to the larger size, resulting in larger effective collision volume. ${ }^{11)}$ In practice, the relative amount of dendritic inclusions should be much larger due to the extremely high local supersaturation degree resulted from the $\mathrm{Al}$ addition. Because of their multi-branching feature, dendritic inclusions can capture each other easily. Consequently, the clustering degree of dendritic inclusions is much higher. The clustering of plate-like inclusions is quite complex. Some plate-like inclusions are very thin and large, mostly exhibiting a hexagonal form, as shown in Fig. 1(b). This type inclusions were occasionally observed in clusters in all samples. On the contrary, some are quite thick and relatively small in size, as shown in Figs. 5(b) and 5(c). A large number of such inclusions were observed in samples L2, HS2, HS3 and HS4. These inclusions frequently cluster edge by edge.

The clustering degrees of different morphologies of alumina inclusions were measured quantitatively for the first time. Different alumina morphologies were found to have quite different clustering tendencies. Since Te affects the morphology distribution of alumina inclusions, the overall clustering degree of alumina inclusions is changed correspondingly. For example, in samples where Te increases the amount of faceted inclusions, which have the highest clustering tendency, the overall clustering degree increases and vice versa. Essentially, the clustering of alumina inclusions results from strong attractive forces between alumina inclusions. It is implied that alumina morphology affects the attractive forces. This will be discussed in the follow-

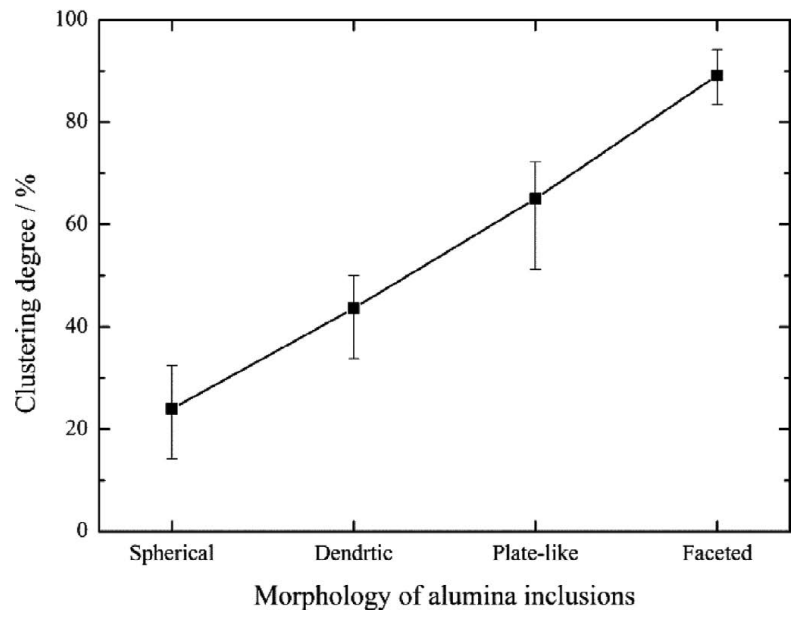

Fig. 4. Average clustering degree as a function of the morphology of alumina inclusions from samples HS1 to HS4.
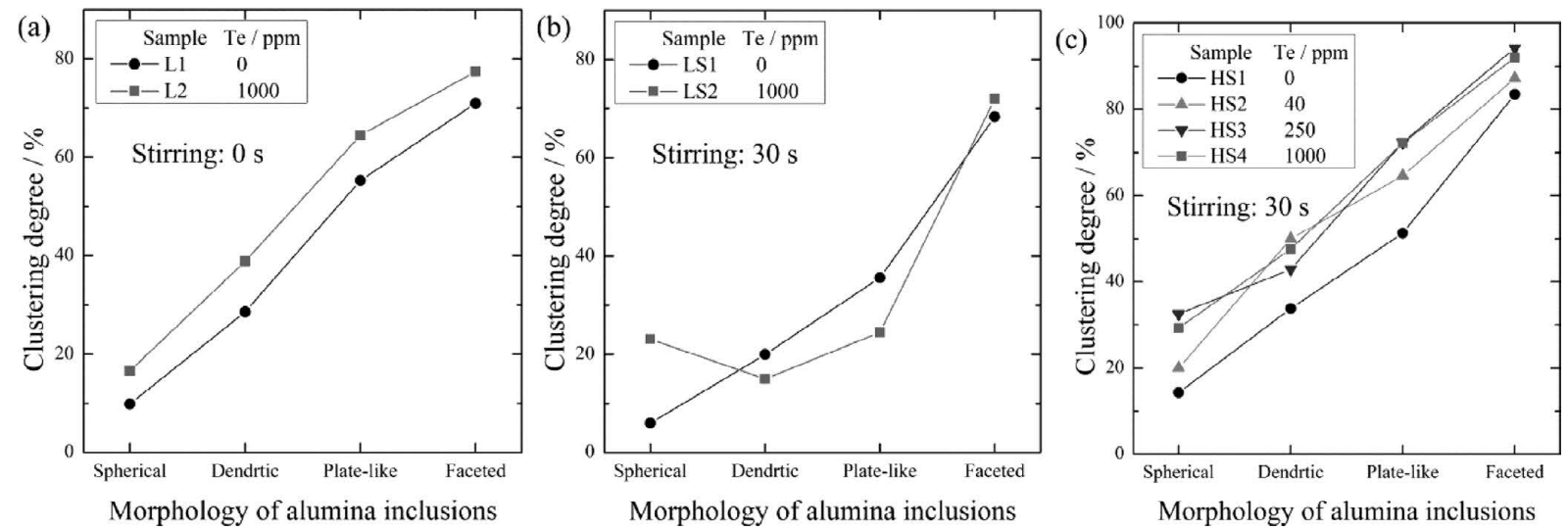

Fig. 3. Clustering degree as a function of the morphology of alumina inclusions. (a) L1 and L2; (b) LS1 and LS2; (c) HS1 to HS4.
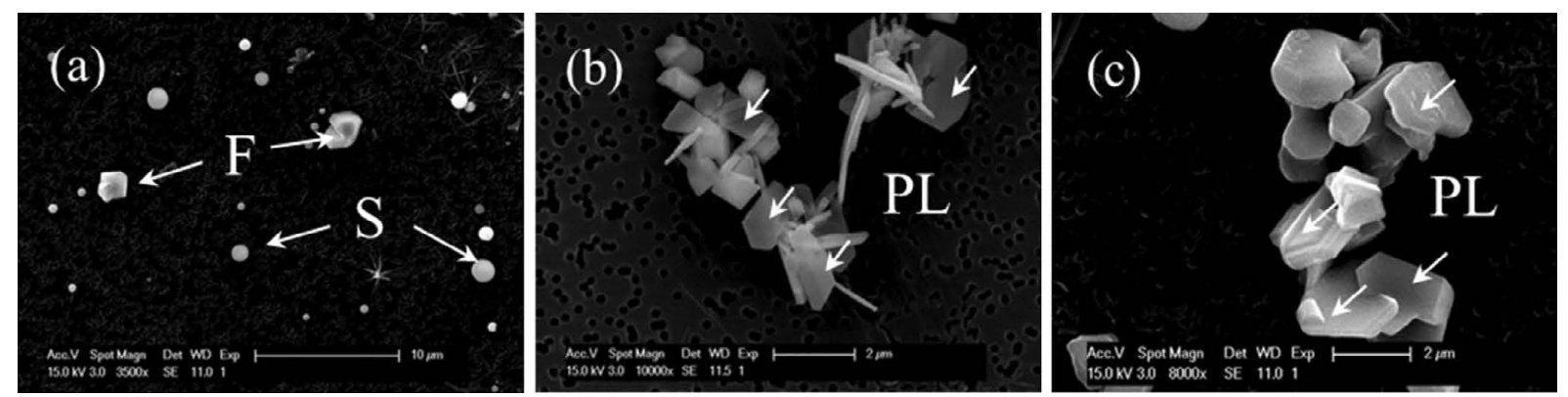

Fig. 5. SEM images of alumina inclusions after acid extraction. ${ }^{29)}$ (a) L1; (b) L2; (c) HS4. 
ing sections.

\subsection{Thermodynamic Considerations on the Clustering of Alumina Inclusions}

3.3.1. Spontaneous Cavitation between Unwetted Particles

High clustering tendency of non-metallic inclusions unwetted by liquid steel indicates that strong attractions exist between the inclusions. Due to the opaque property and the high temperature of liquid steel, attractive forces between non-metallic inclusions in the bulk of liquid steel cannot yet be determined experimentally. However, interactions between unwetted particles in aqueous solutions have been extensively studied, ${ }^{32)}$ revealling the existence of strong attractions of much longer range than the van der Waals force. ${ }^{32-36)}$ Several mechanisms have been proposed to account for these strong attractions: (i) electrostatic interactions; ${ }^{37)}$ (ii) bridging of submicroscopic bubbles adhering to the surface, ${ }^{34,36,38)}$ (iii) disturbed water structure ${ }^{39)}$ and (iv) spontaneous formation of cavities between unwetted surfaces. ${ }^{21,22,40,41)}$ Well-designed experiments have excluded the first two mechanisms. ${ }^{42-44)}$ Of the remaining mechanisms, the proposal that in non-wetting systems the liquid may be spontaneously expelled from the gap between two approaching particles to form a gas cavity, as shown in Fig. $\mathbf{6}$, is the most attractive and has been validated experimentally. ${ }^{45-47)}$ Therefore, the effect of alumina morphology on the clustering of alumina inclusions will be discussed based on the cavitation theory.

For two approaching alumina particles in molten iron, the negative change of Gibbs free energy of an alumina-molten

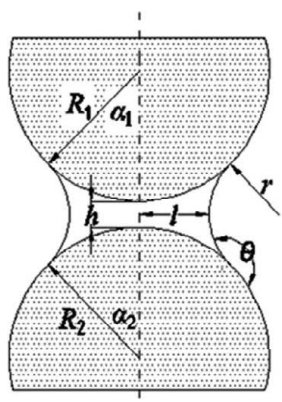

(a) $\mathrm{S}-\mathrm{S}$

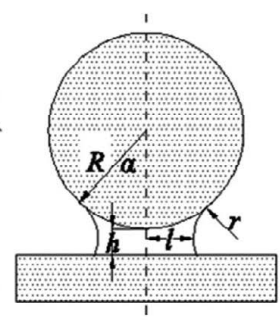

(b) S-P

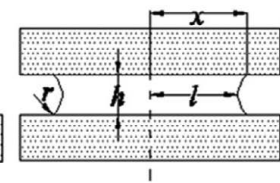

(c) P-P
Fig. 6. Basic geometries of the gas cavities of different contact types. iron interface replaced by an alumina-gas interface, as seen from Eq. (1), is the driving force for the gas cavity formation. ${ }^{21)}$ Once the gas cavity is formed, an attractive force between the two alumina particles develops. This attractive force is the summation of the forces due to the surface tension of molten iron and the pressure drop across the molten iron-gas interface, as expressed in Eq. (2). ${ }^{48)}$

$$
\begin{aligned}
& \gamma_{\mathrm{sg}}-\gamma_{\mathrm{sl}}=\gamma_{\mathrm{lg}} \cdot \cos \theta \\
& F=2 \pi \gamma_{\mathrm{lg}} l+\pi l^{2} \Delta P
\end{aligned}
$$

The pressure drop across the molten iron-gas interface can be calculated with the Laplace-Young equation expressed with Eq. (3).

$$
\Delta P=\gamma_{\lg }(1 / r-1 / l)
$$

where $\gamma_{\mathrm{gg}}$ is the alumina surface tension, $\gamma_{\mathrm{g}}$ is the surface tension of molten iron, $\gamma_{\mathrm{sl}}$ is the alumina-molten iron interfacial energy, $\theta$ is the contact angle, $F$ is the attractive force, $\Delta P$ is the pressure drop, and $l$ and $r$ are the principle radii of the gas cavity, as shown in Fig. 6 .

According to Eqs. (2) and (3), to calculate the attractive force between two alumina particles, the principle radii $l$ and $r$, i.e., the profile of the molten iron-gas interface, should be determined first. Determining exact interface profile requires complex numerical procedures, even for the simple case of two identical spheres. ${ }^{48)}$ Methods of optimizing the interface profile of the cavity confined by two identical spheres are available in literature..$^{21,22,36,49,50)}$ For approximate calculations, the interface is usually described by a piece of a circle. ${ }^{35,48)}$ This method is known as the toroidal approximation and it will be used in the present paper.

By describing the interface profile with a piece of a circle with radius $r$, the principle radii $l$ and $r$ can be expressed as a function of the filling angle $\alpha$. As morphology combinations of two alumina particles are various in liquid steel, here we only consider three cases: sphere and sphere (S-S), sphere and plane (S-P), and plane and plane (P-P). As seen in Fig. 6 , only the particle surface geometry around the contact region affects the gas cavity formation. Therefore, the word "sphere" here indicate the surface around contact region is curved, such as spherical and dendritic inclusions. The word "plane" indicates the surface around contact region is flat, such as faceted and plate-like inclusions. The principle radii $l$ and $r$ are listed in Table 4. In the present paper, the formulas with references are taken from literatures. The formulas

Table 4. The principle radii $l$ and $r$ of a gas cavity for different contact types.

\begin{tabular}{ccc}
\hline \multirow{2}{*}{ Contact types } & \multicolumn{2}{c}{ Principle radii } \\
\cline { 2 - 3 } S-S & $r=-\frac{R_{1}\left(1-\cos \alpha_{1}\right)+R_{2}\left(1-\cos \alpha_{2}\right)+h}{\cos \left(\theta-\alpha_{1}\right)+\cos \left(\theta-\alpha_{2}\right)}$ & $l$ \\
& $r=-\frac{R(1-\cos \alpha)+h}{\cos (\theta-\alpha)+\cos (\theta)}$ & $l=R \sin \alpha-r[1-\sin (\theta-\alpha)]$ \\
S-P $\left.\alpha_{1}-r\right)$ & $r=-\frac{h}{2 \cos \left(\theta^{\prime}\right)}$ & $l=x-r\left(1-\sin \theta^{\prime}\right)$ \\
P-P $P^{36)}$ & $\left.\left.r-\alpha_{1}\right)\right]$ \\
\hline
\end{tabular}

Note: $R, R_{1}$ and $R_{2}$ are the radii of particles; $\alpha, \alpha_{1}$ and $\alpha_{2}$ are the filling angle; $h$ is the separation distance between the two particles; $x$ is the radius of the contact rim (Fig. 6(c)). 
without references are derived by the authors.

The key step of calculating the attractive force $F$ is to determine the filling angle $\alpha$. For rough calculation, the filling angle $\alpha$ can be obtained by inserting the principle radii $l$ and $r$ (as shown in Table 4) into Eq. (3) at constant pressure drop $\Delta P{ }^{26,28)}$ Since this method can not guarantee the formed gas cavity is in thermodynamically stable state, in the present work, the filling angle $\alpha$ was determined by minimizing the Gibbs free energy of the gas cavity, ${ }^{21,49)}$ as expressed with Eq. (4). ${ }^{36)}$

$$
G=P_{\mathrm{o}} V-N k_{\mathrm{B}} T \ln V+\gamma_{\mathrm{lg}} A_{\mathrm{lg}}+\left(\gamma_{\mathrm{sg}}-\gamma_{\mathrm{sl}}\right) \cdot A_{\mathrm{sg}}
$$

where $P_{\mathrm{o}}$ is the pressure in the molten iron, $V$ is the volume of the gas cavity, $N$ is the number of gas molecules inside the cavity, $A_{\lg }$ is the molten iron-gas area, and $A_{\text {sg }}$ is the alumina-gas area.

Expressing $V, A_{\mathrm{lg}}$ and $A_{\mathrm{sg}}$ as a function of the filling angle $\alpha$, the equilibrium value of filling angle $\alpha$ can be obtained from $\mathrm{d} G=f(\alpha)=0$. Then the attractive force $F$ can be calculated. $V$ and $A_{\mathrm{lg}}$ are expressed with Eqs. (5) and (6). The expressions of $A_{\mathrm{sg}}$ are listed in Table $\mathbf{5}$.

$$
\begin{aligned}
& V=\pi \int_{y_{1}}^{y_{2}}\left(r+l-\sqrt{r^{2}-y^{2}}\right)^{2} d y-V_{\mathrm{S}} \\
& A_{\mathrm{lg}}=2 \pi \int_{\theta_{1}}^{\theta_{2}} r(r+l+r \cos (\theta)) d \theta
\end{aligned}
$$

The parameters used in the calculations are listed in Table 6. The number of gas molecules $N$ in the gas cavity is set to 0 , considering that the partial pressure of molten iron and other elements, such as oxygen, is very low. The pressure in the molten iron $P_{\mathrm{o}}$ is set to $1 \mathrm{~atm}$, considering that the depth of the molten iron in the $\mathrm{MgO}$ crucible is very

Table 6. Parameters used in the calculations of the attractive force.

\begin{tabular}{cc}
\hline Parameters & Values \\
\hline Radius of a sphere $R$ & $10 \mu \mathrm{m}$ \\
Radius of a plane (round shape) $R$ & $10 \mu \mathrm{m}$ \\
Number of gas molecules $N$ & 0 \\
Pressure drop $P_{\mathrm{o}}$ & $1 \mathrm{~atm}$ \\
Surface tension of molten iron $\gamma_{\mathrm{gg}}$ & $1.80(\mathrm{~N} / \mathrm{m})^{52)}$ \\
Contact angle $\theta$ & $137\left({ }^{\circ}\right)^{52)}$ \\
The surface tension of Alumina inclusions $\gamma_{\mathrm{sg}}$ & $0.75(\mathrm{~N} / \mathrm{m})^{52)}$ \\
\hline
\end{tabular}

small around $18 \mathrm{~mm}$. The particle radius $R$ is set to $10 \mu \mathrm{m}$ due to the limitation of the toroidal approximation approach. For smaller particle size, the two principle radii $l$ and $r$ are almost at the same size. Thus, it is not possible to keep the pressure drop $\Delta P$ approximately constant. ${ }^{51)}$ For the S-S and S-P contact types, the contact angle at the three-phase line equals the bulk contact angle when the Gibbs free energy of the cavity is minimized. ${ }^{36)}$ It is therefore kept constant during calculation. For the P-P contact type, the contact angle at the three-phase line is allowed to vary, which can be understood from Fig. 7(c) in section 3.3.2. Instead, the pressure drop $\Delta P$ is kept constant.

\subsubsection{Gibbs Free Energy of A Gas Cavity between Two Alumina Particles}

Figure 7 shows the calculated Gibbs free energy of a gas cavity as a function of the filling angle $\alpha$ or the cavity radius $l$ at different separation distances. For the contact types S-S (Fig. 7(a)) and S-P (Fig. 7(b)), at small separation distance, the Gibbs free energy always has a minimum value, where the gas cavity is thermodynamically stable. This implies that a gas cavity forms spontaneously when the distance between two approaching alumina particles is small enough. Increasing the separation distance makes the gas cavity thermodynamically metastable and finally unstable, which means that the gas cavity will break and the attractive force will disappear. For the contact type P-P (Fig. 7(c)), the Gibbs free

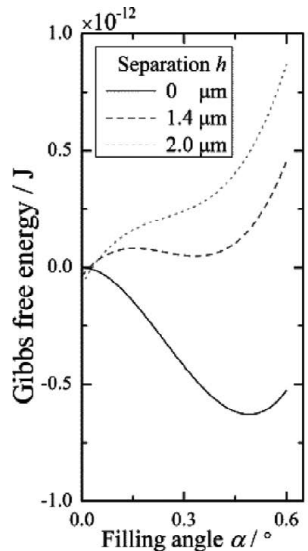

(a) S-S

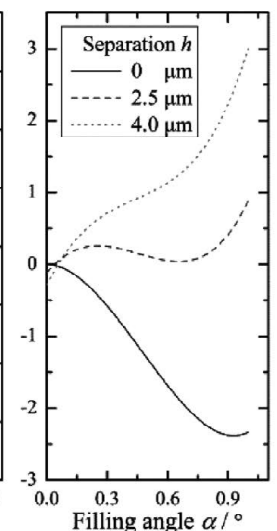

(b) S-P

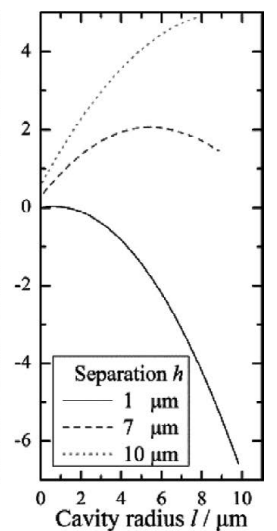

(c) P-P
Fig. 7. Variation of Gibbs free energy of a gas cavity with filling angle $\alpha$ or cavity radius $l$ at different separation distances

\begin{tabular}{|c|c|c|c|c|c|c|}
\hline \multirow{2}{*}{ Contact types } & \multirow{2}{*}{$\begin{array}{l}\text { Alumina-gas area } \\
\qquad A_{\mathrm{sg}}\end{array}$} & \multicolumn{2}{|c|}{ Molten iron-gas area $A_{\text {lg }}$} & \multicolumn{3}{|c|}{ Volume of gas cavity $V$} \\
\hline & & $\theta_{1}$ & $\theta_{2}$ & $y_{1}$ & $y_{2}$ & $V_{\mathrm{S}}$ \\
\hline S-S & $\begin{array}{c}2 \pi R_{1}^{2}\left(1-\cos \alpha_{1}\right)+ \\
2 \pi R_{2}^{2}\left(1-\cos \alpha_{2}\right)\end{array}$ & $\frac{3 \pi}{2}-\theta+\alpha_{1}$ & $\frac{\pi}{2}+\theta-\alpha_{2}$ & $r \cos \left(\theta-\alpha_{2}\right)$ & $r \cos \left(\alpha_{1}-\theta\right)$ & $\begin{array}{c}\frac{\pi}{3} R_{1}^{3}\left(2+\cos \alpha_{1}\right) \cdot\left(1-\cos \alpha_{1}\right)^{2} \\
\frac{\pi}{3} R_{2}^{3}+\left(2+\cos \alpha_{2}\right) \cdot\left(1-\cos \alpha_{2}\right)^{2}\end{array}$ \\
\hline S-P & $\begin{array}{c}2 \pi R_{1}^{2}(1-\cos \alpha) \\
+\pi(r+l-r \sin \theta)^{2}\end{array}$ & $\frac{3 \pi}{2}-\theta+\alpha$ & $\frac{\pi}{2}+\theta$ & $r \cos \theta$ & $r \cos (\alpha-\theta)$ & $\frac{\pi}{3} R^{3}(2+\cos \alpha) \cdot(1-\cos \alpha)^{2}$ \\
\hline P-P & $2 \pi x^{2}$ & $\frac{3 \pi}{2}-\theta^{\prime}$ & $\frac{\pi}{2}+\theta^{\prime}$ & $r \cos \theta^{\prime}$ & $-r \cos \theta^{\prime}$ & 0 \\
\hline
\end{tabular}
for different contact types. (a): S-S; (b): S-P; (c): P-P.

Table 5. $\quad A_{\mathrm{sg}}, A_{\lg }$ and $V$ for different contact types. 
energy varies differently. When the separation distance is small, the Gibbs free energy has no minimum value, which means that the gas cavity can grow unlimitedly. In practice, the growth of the gas cavity is limited by the particle size. With the increase of separation distance, the cavity becomes metastable and finally unstable.

\subsubsection{Attractive Force and Rupture Energy}

The calculated attractive force between two alumina particles as a function of the separation distance is shown in Fig. 8(a). The P-P type has the strongest attractive force and the longest critical separation distance $(9.20 \mu \mathrm{m})$. The S-S type has the weakest attractive force and the shortest critical separation distance $(1.56 \mu \mathrm{m})$, which is still approximately two orders of magnitude longer than the van der Waals force (Fig. 9(c)). To the best of our knowledge, this is the first quantitative calculation of the attractive force between alumina inclusions in liquid steel based on spontaneous cavitation theory, especially focusing on the effect of morphology. The dependence of forces on particle shape is known as Derjaguin's approximation, according to which the force due to atomic or molecular interactions (for example, van der Waals force) of a S-S type is half of that of a S-P type. ${ }^{33)}$ In the present work, the ratio of the attractive force between the S-S type and S-P type is calculated to be 0.52 when two particles contact. Figure 8(b) shows the attractive forces arising from surface tension and pressure drop, separately, for the S-S contact type. As seen, the force due to surface tension dominates.

The area under the force-separation distance curve is a measure for the energy required to rupture the gas cavity. The larger the rupture energy, the more difficult it is to separate alumina particles. The rupture energies of different contact types are shown in Fig. 8(c). The rupture energy of P-P type is 16.4 times and 4.2 times as large as that of S-S type and S-P type, respectively. This suggests that non-metallic inclusions with large flat faces, such as faceted and plate-like inclusions, should form clusters more easily than spherical inclusions. This explains why the clustering degree of faceted alumina inclusions is much larger than that of spherical inclusions. The ratio of average cluster- ing degree between faceted and spherical inclusions in tests HS1 to HS4 is calculated to be 3.7. This value is very close to the rupture energy ratio between P-P type and S-P type. As the relative amount of spherical inclusions is very low (3.1-8.3\%), spherical inclusions mainly cluster with faceted inclusions. The consistency further confirms the occurrence of spontaneous cavitation between approaching alumina inclusions. Moreover, break-up of large clusters in liquid steel depends on the balance between the force due to fluid flow and the attractive force between inclusions. The calculation suggests that faceted inclusions can form large clusters more easily. This is beneficial to the removal of inclusions, as confirmed by Horigome et al. ${ }^{53)}$

\subsubsection{Comparison with the Capillary Force on Melt Sur- face and the van der Waals Force}

To have a better understanding of the attractive force due to gas cavity formation, we compare this force with the capillary force on melt surface and the van der Waals force, as shown in Fig. 9. The van der Waals force between two spherical particles is expressed with Eq. (7). ${ }^{54}$

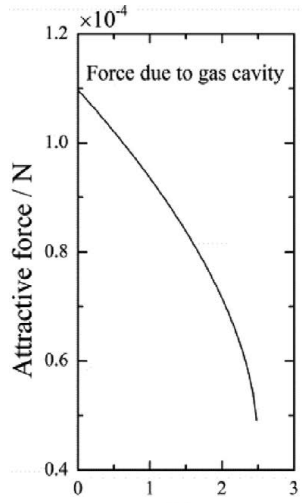

(a)

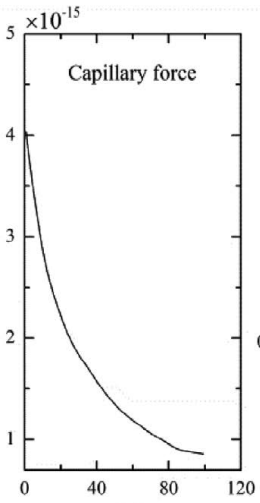

(b)

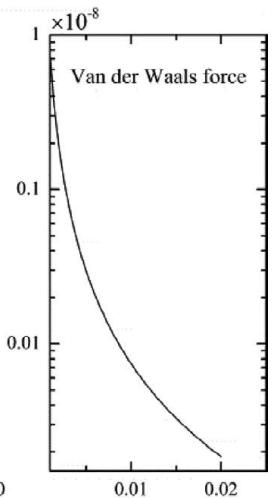

(c)
Separation distance $/ \mu \mathrm{m}$

Fig. 9. Comparison of different attractive forces between two spherical alumina particles. (a) attractive force due to gas cavity formation; (b) capillary force and (c) van der Waals force.

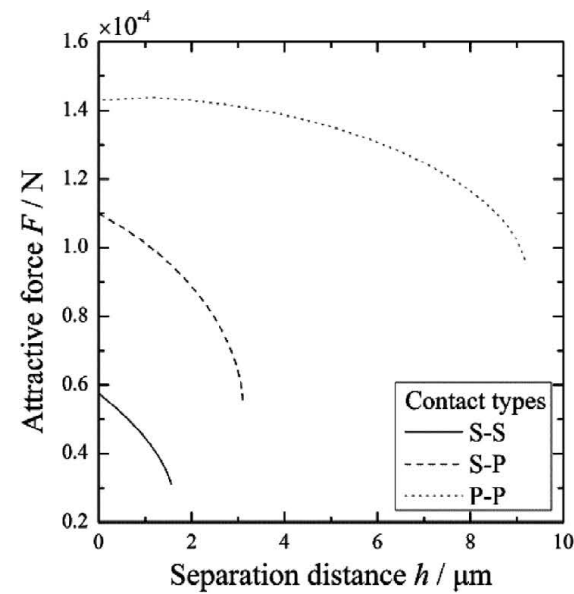

(a)

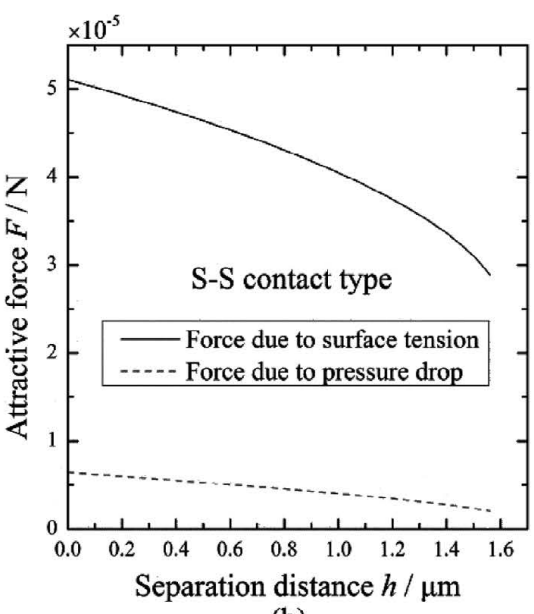

(b)

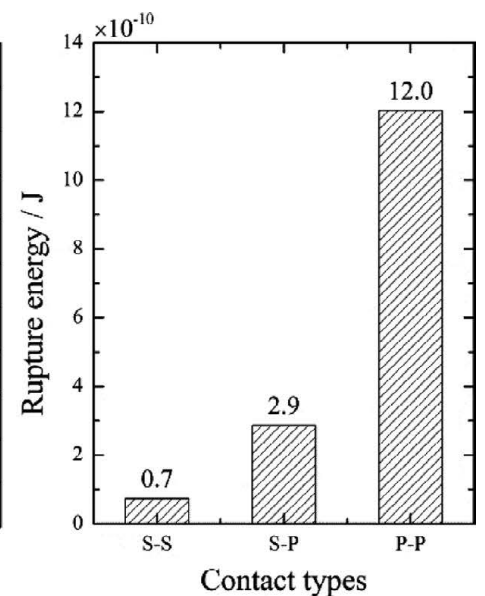

(c)

Fig. 8. Calculated attractive force and rupture energy for different contact types. (a) and (b) attractive force; (c) rupture energy. 


$$
F_{\mathrm{VDW}}=\frac{A_{121}}{6 h^{2}} \cdot \frac{R_{1} R_{2}}{\left(R_{1}+R_{2}\right)}
$$

where $A_{121}$ is the Hamaker constant, $h$ is the inter-particle distance and $R_{1}$ and $R_{2}$ are the radii of spherical particles. As calculation of the capillary force between two particles on melt surface is quite complex, the capillary force is taken directly from Ref. 18). For consistency, parameters used in the comparison are taken from the same reference. They are listed in Table 7.

As seen in the figure, the attractive force due to gas cavity formation is the strongest, $10^{-5}$ to $10^{-4} \mathrm{~N}$, with acting length around $2.5 \mu \mathrm{m}$. The capillary force is the weakest, $10^{-16}$ to $10^{-15} \mathrm{~N}$, however, with the longest acting length up to $100 \mu \mathrm{m}$. This is confirmed by in-situ observation by CSLM $^{15)}$ The van der Waals force, $10^{-11}$ to $10^{-8} \mathrm{~N}$, is several orders of magnitude stronger than the capillary force, however, with the shortest acting length. Different attractive forces play different roles in the clustering of non-metallic inclusions in liquid steel. On the surface of liquid steel, unwetted inclusions apart tens of micrometers are observed to move to each other. The capillary force is believed to be responsible for the long-range attraction on melt surface. ${ }^{15)}$ In the bulk of liquid steel, the capillary force disappears due to the absence of gas-liquid-solid interfaces. As clustering occurs mainly among unwetted inclusions, the van der Waals force can be ruled out. At present, the strong attractive force due to gas cavity formation seems to be the only reasonable explanation for the clustering among unwetted inclusions in the bulk of liquid steel.

\subsubsection{Influence of Surface Tension, Contact Angle and} Particle Size on the Attractive Force

To elucidate the factors that influence the attractive force, we take surface tension, contact angle and particle size into account. Figure 10(a) shows that the rupture energy between two identical spherical alumina particles increases linearly with increasing surface tension of the melt. Figure 10(b) shows that the rupture energy increases slowly with increasing contact angle to $120^{\circ}$, thereafter it increases sharply with contact angle. This can explain why the clustering of $\mathrm{Al}_{2} \mathrm{O}_{3}$ is more severe compared with $\left.\mathrm{SiO}_{2}\left(115^{\circ}\right),{ }^{55}\right)$ $\mathrm{ZrO}_{2}\left(122^{\circ}\right)^{52)}$ and $\mathrm{MgO}\left(125^{\circ}\right) .^{52)}$

According to Fig. 10(a), clustering of non-metallic inclusions in liquid steel might be weakened by adding surfactants, which decrease the surface tension of liquid steel. In practice, however, this effect is not obvious as surfactants increase the contact angle simultaneously. An example of Te addition affecting the rupture energy between two spherical alumina particles in molten iron is shown in Fig. 11. The

Table 7. Parameters used in the comparison of different forces.

\begin{tabular}{cc}
\hline Parameters & Values \\
\hline Surface tension of molten iron $\gamma_{g}$ & $1.80(\mathrm{~N} / \mathrm{m})^{18)}$ \\
Contact angle $\theta$ & $133\left({ }^{\circ}\right)^{18)}$ \\
Hamaker constant $a_{121}$ & $0.45 \times 10^{-20}(\mathrm{~J})^{27)}$ \\
Alumina particle radius $R_{1}=R_{2}$ & $20 \mu \mathrm{m}^{18)}$ \\
\hline
\end{tabular}

parameters used are listed in Table 8. The rupture energy increases abruptly with increasing Te addition from 0 to 40 ppm, thereafter decreases gradually with Te addition.

Figure 12 shows that the rupture energy between two alumina particles of S-P type increases linearly with increasing the size of the spherical particle. The size of the plane is assumed to be infinite. This suggests that large particles are more likely to form clusters, as confirmed in our previ-

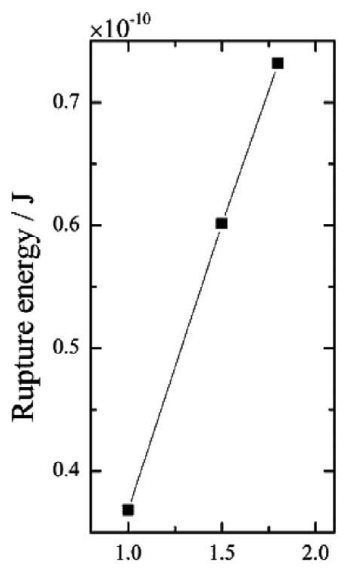

Surface tension $\gamma_{1 \mathrm{~g}} / \mathrm{N} \cdot \mathrm{m}^{-1}$ (a)

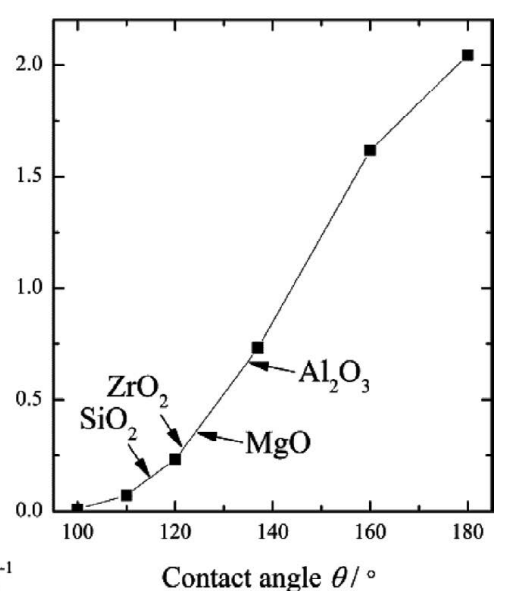

(b)
Fig. 10. Effects of surface tension and contact angle on the rupture energy between two identical spherical alumina particles with $R_{1}=R_{2}=10 \mu \mathrm{m}$. (a) influence of surface tension at $\alpha=137^{\circ}$; (b) influence of contact angle at $\gamma_{\mathrm{g}}=1.80$ $\mathrm{N} / \mathrm{m}$

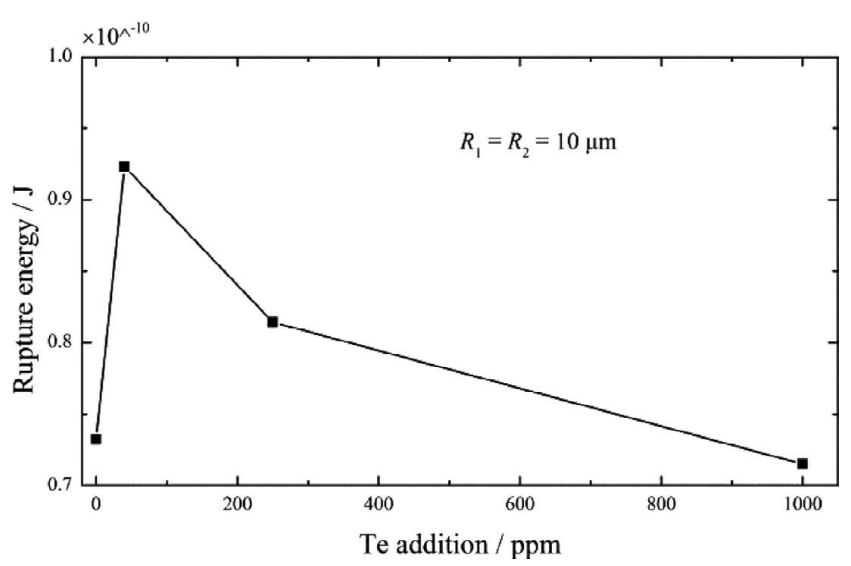

Fig. 11. Effect of Te addition on the rupture energy between two spherical alumina particles in molten iron.

Table 8. Interfacial properties used in the calculation of rupture energy. ${ }^{56)}$

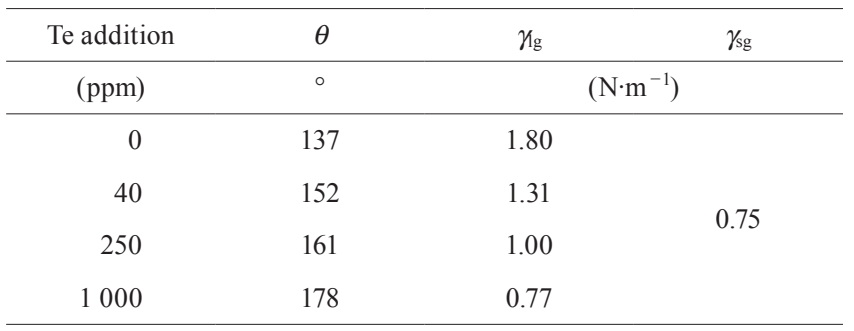


ous paper. ${ }^{29)}$

\subsection{Clustering of Plate-like Alumina Inclusions}

Theoretical calculations in section 3.3.5 suggest that plate-like alumina inclusions should form clusters more easily than faceted inclusions as their large flat faces can generate stronger attractive force. However, this is inconsistent with experimental results of the clustering degree of plate-like alumina inclusions, which is approximately $25 \%$ lower than that of faceted inclusions in samples HS1 to HS4. Plate-like alumina inclusions exhibit a platy $\{222\}$ habit. ${ }^{57,58)}$ To account for this discrepancy, we think that molten iron wets plate-like inclusions better. This is confirmed by literature. Table 9 summarizes literature data

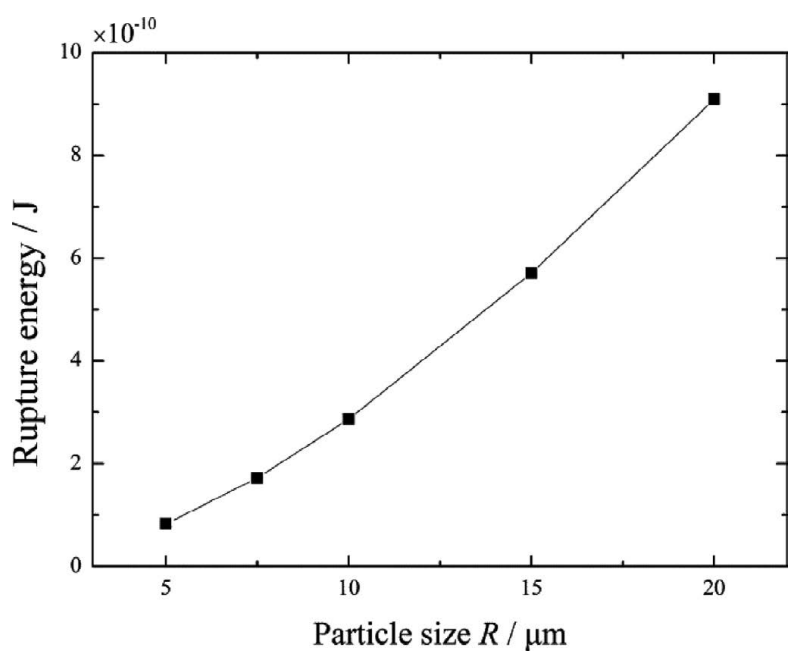

Fig. 12. Influence of particle size on the rupture energy between two alumina particles of S-P type at $\alpha=137^{\circ}$ and $\gamma_{\mathrm{g}}=1.80$ $\mathrm{N} / \mathrm{m}$.

Table 9. Contact angle of molten iron on an alumina substrate.

\begin{tabular}{ccccc}
\hline Contact angle $/{ }^{\circ}$ & Substrate & Temperature $/ \mathrm{K}$ & Atmos. & Ref. \\
\hline 132 & Polycrystalline & 1873 & $\mathrm{Ar}$ & $59)$ \\
137 & Polycrystalline & 1873 & $\mathrm{H}_{2}$ & $56)$ \\
145 & Polycrystalline & 1873 & $\mathrm{H}_{2}$ & $60)$ \\
$105-118$ & Single crystal & 1873 & $\mathrm{CO}$ & $61)$ \\
104 & (222) face & 1873 & $\mathrm{H}_{2}-\mathrm{Ar}$ & $62)$ \\
108 & (222) face & 1973 & $\mathrm{Ar}$ & $63)$ \\
\hline
\end{tabular}

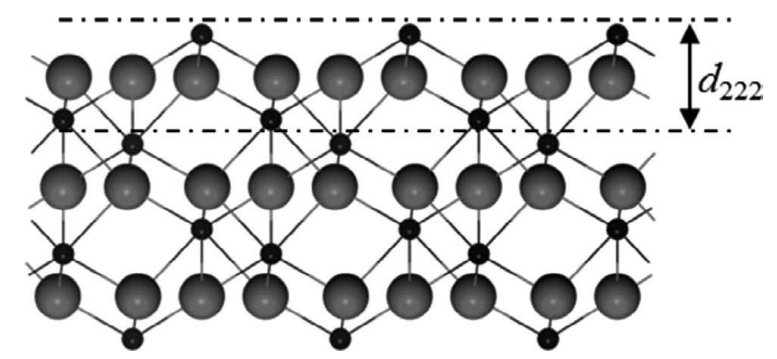

Fig. 13. Side view of (222) crystal face of $\alpha-\mathrm{Al}_{2} \mathrm{O}_{3}$ along $<0 \overline{1} 1>$. $\mathrm{O}$ : large red spheres; $\mathrm{Al}$ : small black spheres. on the measured contact angle between molten iron and an alumina substrate.

The contact angle between the (222) face and molten iron is approximate $30^{\circ}$ smaller than that between polycrystalline alumina substrate and molten iron. Due to the small contact angle of $104-108^{\circ}$, a weak attractive force between a platelike alumina particle and a particle of any shape is predicted. The small contact angle of molten iron on a (222) face is due to its special surface structure. ${ }^{62)}$ As shown in Fig. 13, the (222) face has the highest density of unsaturated bonds, i.e., one broken bond for each $\mathrm{O}$ atom and three broken bonds for each $\mathrm{Al}$ atom. ${ }^{64)}$ Due to such a surface structure, strong adsorption of $\mathrm{Fe}$ atoms is expected. This can improve the wettability of molten iron on (222) face.

\section{Conclusion}

The effect of alumina morphology on the clustering of alumina inclusions in molten iron was studied. The results obtained are as follows:

(1) Te addition modifies the morphology of alumina inclusions. Te tends to make the morphology of alumina inclusions evolve from dendritic and spherical, via faceted, to plate-like. Stirring strengthens the effectiveness of Te on the morphological modification of alumina inclusions, resulting in more plate-like inclusions. Increasing the supersaturation degree weakens the effectiveness of Te.

(2) Alumina morphology affects alumina clustering degree. The clustering degree increases in the order of spherical, dendritic, plate-like and faceted inclusions.

(3) Attractive force between two alumina inclusions with different shape combinations, i.e., S-S, S-P and P-P, were calculated based on the spontaneous cavitation theory. S-S type has the smallest attractive force and the shortest acting length; P-P type has the largest attractive force and the longest acting length. This can explain why spherical inclusions have the smallest clustering degree.

(4) The attractive force due to gas cavity formation was compared with the capillary force on melt surface and the van der Waals force. The attractive force due to gas cavity formation is the strongest in magnitude while the capillary force is the weakest.

(5) Plate-like alumina inclusions have $25 \%$ lower clustering degree, compared with faceted inclusions. This is due to that plate-like inclusions have better wettability by molten iron.

\section{Acknowledgement}

The authors thank the China Scholarship Council (CSC) for financial support (File No. 201206080011).

\section{REFERENCES}

1) A. Nicholson and T. Gladman: Ironmaking Steelmaking, 13 (1986), 53.

2) H. V. Atkinson and G. Shi: Prog. Mater. Sci., 48 (2003), 457.

3) S. X. Li: Int. Mater. Rev., 57 (2012), 92.

4) K. Rackers and B. Thomas: 78th Steelmaking Conf. Proc., ISS, Warrendale, PA, (1995), 723.

5) J. M. A. Geldenhuis and P. C. Pistorius: Ironmaking Steelmaking, 27 (2000), 442.

6) L. Holappa, M. Hamalainen, M. Liukkonen and M. Lind: Ironmaking Steelmaking, 30 (2003), 111.

7) H. Ooi, M. Sekine and G. Kasai: Tetsu-to-Hagané, 59 (1973), 1078. 
8) Y. Murakata, M. G. Sung, K. Sassa and S. Asai: ISIJ Int., 47 (2007), 633.

9) K. Fuchigami, M. Wakoh, N. Imamura, K. Endoh, A. Kiyose and I Sawada: Tetsu-to-Hagané, 85 (1999), 368.

10) H. Tozawa, Y. Kato, K. Sorimachi and T. Nakanishi: ISIJ Int., 39 (1999), 426.

11) T. Braun, J. Elliott and M. Flemings: Metall. Trans. B, 10 (1979), 171.

12) M. X. Guo and H. Suito: ISIJ Int., 39 (1999), 678.

13) Y. Kang, B. Sahebkar, P. R. Scheller, K. Morita and D. Sichen: Metall. Mater. Trans. B, 42 (2011), 522.

14) B. Coletti, S. Vantilt, B. Blanpain and S. Sridhar: Metall. Mater. Trans. B, 34 (2003), 533

15) H. B. Yin, H. Shibata, T. Emi and M. Suzuki: ISIJ Int., 37 (1997), 936.

16) H. Shibata, H. Yin and T. Emi: Philos. Trans. R. Soc. Lond., A, 356 (1998), 957.

17) K. Nakajima and S. Mizoguchi: Metall. Mater. Trans. B, 32 (2001), 629.

18) H. Yin, H. Shibata, T. Emi and J. Kim: 2nd HTC, Foundry Research Institute, Cracow, Poland, (1997), 380.

19) S. H. Lee, C. Tse, K. W. Yi, P. Misra, V. Chevrier, C. Orrling, S. Sridhar and A. W. Cramb: J. Non-Cryst. Solids, 282 (2001), 41.

20) P. A. Kralchevsky and K. Nagayama: Adv. Colloid Interface Sci., 85 (2000), 145.

21) V. S. Yushchenko, V. V. Yaminsky and E. D. Shchukin: J. Colloid Interface Sci., 96 (1983), 307.

22) V. V. Yaminsky, V. S. Yushchenko, E. A. Amelina and E. D. Shchukin: J. Colloid Interface Sci., 96 (1983), 301.

23) M. Cournil, F. Gruy, P. Gardin and H. Saint-Raymond: Phys. Status Solidi A, 189 (2002), 159.

24) F. Gruy, M. Cournil and P. Cugniet: J. Colloid Interface Sci., 284 (2005), 548.

25) M. Nakamoto, T. Tanaka, M. Suzuki, K. Taguchi, Y. Tsukaguchi and T. Yamamoto: ISIJ Int., 54 (2014), 1195.

26) K. Sasai and Y. Mizukami: ISIJ Int., 41 (2001), 1331.

27) T. Mizoguchi, Y. Ueshima, M. Sugiyama and K. Mizukami: ISIJ Int., 53 (2013), 639

28) K. Sasai: ISIJ Int., 54 (2014), 2780.

29) L. Zheng, A. Malfliet, P. Wollants, B. Blanpain and M. Guo: ISIJ Int., 55 (2015), 1891.

30) R. Dekkers, B. Blanpain and P. Wollants: Metall. Mater. Trans. B, 34 (2003), 161.

31) Z. Berkovitch-Yellin: J. Am. Chem. Soc., 107 (1985), 8239.

32) J. Israelachvili and R. Pashley: Nature, 300 (1982), 341.

33) H. K. Christenson and P. M. Claesson: Adv. Colloid Interface Sci., 91 (2001), 391
34) N. Ishida, M. Sakamoto, M. Miyahara and K. Higashitani: Langmuir, 16 (2000), 5681

35) M. A. Hampton and A. V. Nguyen: J. Colloid Interface Sci., 333 (2009), 800

36) J. L. Parker, P. M. Claesson and P. Attard: J. Phys. Chem., 98 (1994), 8468.

37) R. Podgornik: J. Chem. Phys., 91 (1989), 5840

38) A. Carambassis, L. C. Jonker, P. Attard and M. W. Rutland: Phys. Rev. Lett., 80 (1998), 5357.

39) V. V. Yaminsky and B. W. Ninham: Langmuir, 9 (1993), 3618.

40) S. Singh, J. Houston, F. van Swol and C. J. Brinker: Nature, 442 (2006), 526.

41) D. Bratko, R. A. Curtis, H. W. Blanch and J. M. Prausnitz: J. Chem. Phys., 115 (2001), 3873.

42) E. E. Meyer, Q. Lin and J. N. Israelachvili: Langmuir, 21 (2005), 256.

43) J. L. Parker and P. M. Claesson: Langmuir, 10 (1994), 635.

44) V. S. J. Craig, B. W. Ninham and R. M. Pashley: Langmuir, 14 (1998), 3326.

45) H. K. Christenson and P. M. Claesson: Science, 239 (1988), 390.

46) R. M. Pashley, P. M. Mcguiggan, B. W. Ninham and D. F. Evans: Science, 229 (1985), 1088.

47) J. Wood and R. Sharma: Langmuir, 11 (1995), 4797.

48) S. J. R. Simons, J. P. K. Seville and M. J. Adams: Chem. Eng. Sci., 49 (1994), 2331.

49) P. Attard: Langmuir, 16 (2000), 4455.

50) J. C. Eriksson and S. Ljunggren: Langmuir, 11 (1995), 2325.

51) M. Dormann and H. J. Schmid: Langmuir, 30 (2014), 1055.

52) K. Nogi and K. Ogino: Can. Metall. Q., 22 (1983), 19.

53) T. Horigome, K. Shinmyo, T. Sato and M. Wakabayashi: Tetsu-toHagané, 59 (1973), 816.

54) M. H. Korayem and Z. Rastegar: Lat. Am. Appl. Res., 43 (2013), 313.

55) P. Kozakevi and M. Olette: Rev. Metall., 68 (1971), 635.

56) K. Ogino, K. Nogi and O. Yamase: Trans. Iron Steel Inst. Jpn., 23 (1983), 234.

57) K. Watanabe, Y. Sumiyoshi and I. Sunagawa: J. Cryst. Growth, 42 (1977), 293.

58) K. Watanabe and I. Sunagawa: J. Cryst. Growth, 65 (1983), 568.

59) E. Kapilashrami, A. Jakobsson, A. K. Lahiri and S. Seetharaman: Metall. Mater. Trans. B, 34 (2003), 193.

60) K. Nakashima, K. Takihira, K. Mori and N. Shinozaki: Mater. Trans. JIM, 33 (1992), 918.

61) S. Ueda, H. Shi, X. Jiang, H. Shibata and A. W. Cramb: Metall. Mater. Trans. B, 34 (2003), 503.

62) J. Lee and S. Cho: Kor. J. Mater. Res., 18 (2007), 18.

63) E. Ricci, D. Giuranno and N. Sobczak: J. Mater. Eng. Perform., 22 (2013), 3381.

64) P. Hartman: J. Cryst. Growth, 49 (1980), 166. 